\title{
Diagnostic Value of Video Head Impulse Test for Patients with Benign Paroxysmal Positional Vertigo
}

\author{
Özlem Yüksel Coşar ${ }^{1}$, Akif İşlek ${ }^{2}$, and Hale Aslan ${ }^{3}$ \\ ${ }^{1}$ Dr Ersin Arslan Training and Research Hospital \\ ${ }^{2}$ Nusaybin State Hospital \\ ${ }^{3}$ Izmir Katip Celebi University
}

October 15, 2020

\begin{abstract}
Objectives: This study aims to investigate the value of Video head impulse test (vHIT) findings in peripheral vertigo diseases, especially in Benign paroxysmal positional vertigo (BPPV) in the acute period. Design: A prospective study was designed. Setting: Otorhinolaryngology and Audiology Clinic of the tertiary university, October 2017-September 2018. Participants: Patients diagnosed with peripheral vertigo included prospectively and consecutively in the study. Main outcome measures: Demographic characteristics of the patients and pathological SCC detected by examination and diagnostic maneuver for BPPV patients were recorded. Vestibulo-ocular reflex (VOR) gain was calculated in all patients and all six SCCs. Two sub-study groups were formed according to age $(<50$ or $>50)$. Also VOR gain detected for BPPV, Vestibular neuritis $(\mathrm{VN})$, and Meniere's disease (MD). Results: 74 (82.2\%) of the patients were Benign paroxysmal positional vertigo (BPPV), eight (8.9\%) of patients were vestibular neuritis $(\mathrm{VN})$, and eight $(8.9 \%)$ of them were Meniere's disease (MD). Pathological deficiency of VOR for SCCs was detected in 49 of $74(66.2 \%)$ patients with BPPV, all eight patients with VN (100\%), and four of eight patients with Meniere's disease (50\%). The sensitivity and specificity calculated as $55.4 \%$ and $81.2 \%$ for BPPV patients. Conclusions: vHIT is a new protocol and provides objective findings for SCC functions in patients with peripheral vertigo. vHIT can be performed faster and easier than the caloric stimulation test for VN diagnosis with comparable results. However, vHIT does not provide sufficient results for BPPV to distinguish from other peripheral vestibular system diseases.
\end{abstract}

\section{Key Points}

1- vHIT provides objective findings for SCC functions.

2- vHIT can be performed faster and easier than the caloric stimulation test.

3- The test may help for differential diagnosis of acute vertigo.

4- vHIT is recommended as an important diagnostic test for Vestibular neuritis and Meniere's disease.

5- vHIT does not provide specific information for benign paroxysmal positional vertigo.

\section{Introduction}

Benign paroxysmal positional vertigo (BPPV) is the most common pathogenesis of peripheral vertigo, and semicircular canal (SCC) involvement and underlying pathophysiologic mechanisms determine the direction and characteristics of nystagmus [1]. Meniere's disease is an internal ear disease that courses with spontaneous vertigo attacks, fluctuating sensorineural hearing loss, ear fullness, and tinnitus [2]. Vestibular neuritis (VN) is selective, acute, or subacute inflammation of the vestibular nerve. Mostly superior branch of vestibular nerve is affected and dysfunction of these fibers arises [3]. 
Video head impulse test (vHIT) is a new and important physiological test to detect the gain of the vestibuloocular reflex (VOR) for each semicircular and finds out overt and covert catch-up saccades with the head impulse and gaze deviation $[4,5]$. Although the caloric test is the gold standard in the diagnosis of peripheral vestibular diseases, vHIT is a simple and fast test, it does not cause nausea and discomfort in the patient, and provides a separate evaluation for each SCC [5,6]. While vHIT is recommended as an important diagnostic test for $\mathrm{VN}$ and $\mathrm{MD}$ in the literature, it has been reported as unnecessary and unfavorable in BPPV patients[5, 7]. However, there are a few clinical studies not including the detailed analysis of BPPV patient's findings. The information for vHIT results for BPPV in the literature was generally derived from the results of a group of patients with acute or peripheral vertigo.

\section{Material and Methods}

Patients were included prospectively and consecutively in the study. The study was conducted in the Otorhinolaryngology and Audiology Clinic of the tertiary university hospital between October 2017 and September 2018. Demographic characteristics of the patients and pathological SCC detected by examination and diagnostic maneuver for BPPV patients were recorded. VOR gain was calculated in all patients and all six SCCs. Two sub-study groups were formed according to age $(<50$ or $>50)$. The study was prepared with the reporting guideline of the STROBE checklist.

Inclusion: Only patients with complaints of acute onset vertigo ( $<5$ days) were included in the study. The diagnosis of BPPV was made by examination, clinical history, clinical head impulse test (cHIT), and Dix Hallpike maneuver for vertical SCCs and examination, clinical history, clinical head impulse test (cHIT), and roll test for the lateral SCC. Vestibular Neuritis was diagnosed by medical history, physical examination, and caloric tests. The final diagnosis in VN patients was made by caloric test (irrigation of each ear with water at a temperature of 30 and $44{ }^{\circ} \mathrm{C}$ ) A $20 \%$ difference calculated by Jongkees formula between the ears was accepted a pathological response. Meniere's disease (MD) diagnosis was made in accordance with the diagnostic criteria of AAO-HNS Committee on Hearing and Equilibrium Meniere's Disease [8].

Exclusion: The patients with immunocompromised conditions, autoimmune system diseases, malignancy, history of ear surgery, history of migraine, and history of antivertigo drugs in the last two weeks excluded from the study. Patients who did not receive the three diagnoses included in this study during or after the study protocol and the findings of these patients were not included in the final analysis.

vHIT protocol : Initially, the physiological lower limit of VOR gain was determined in 20 healthy participants and recorded by the instrument (Fig. 1). The accepted lower value of VOR gain determined as 0.9 for the lateral SCCs and 0.7 for vertical SCCs. vHIT protocol of Micromedical Technologies Company's VisualEyes Four-Canal-Videonystagmography (VNG) instrument was used for the test. This test consists of 3 parts. The first part is Lateral (Left Lateral: LL - Right Lateral: RL) test which is used for examination of lateral SCCs (LSCC). The second and third parts are RALP (Right Anterior : RA - Left Posterior: LP) and LARP (Left Anterior: LA - Right Posterior: RP) tests which are used for examination of vertical canals. During the test, fast pushing force is applied to patient's head with approximately 20deg-30deg angle by audiologist/physician according to SCCs canal axis. Patients were asked to unloose her/his head, not to contract trapezius muscle and not to turn her/his eyes away from the point adjusted on the wall in 1.5-meterdistance from the patient. For each canal, at least 10-15 head push movements were applied. Lateral, RALP, LARP parameters of all patients were evaluated and VOR gains for six SCCs were determined separately. As a result of VHIT implemented on 20 normal patients, standard VOR values were priorly recorded by the instrument. Normal standard lower value of VOR was accepted as 0.92 for lateral canal and 0.74 for vertical canals. Based on these values VOR gain values of patients were examined.

Statistical Analysis: Data for the study were deposited with an electronic file. The findings analyzed by the SPSS 22.0 (SPSS Inc., Armonk, NY) software system. The normality assumption was measured by the Kolmogorov Smirnov test. Categorical variables were compared with the chi-square test, and scale variables were compared with the Kruskal-Wallis test. Sensitivity and specificity were calculated for all and each SCCs causing BPPV. Results with a significance coefficient of $\mathrm{p}>0.05$ will be considered statistically significant. 
Ethics Committee Approval: Verbal and written consent was obtained after all participants were informed. All procedures performed in studies involving human participants were following the 1964 Helsinki Declaration and its later amendments or comparable ethical standards.

\section{Results}

90 patients were included in the study. $74(82.2 \%)$ of the patients were Benign paroxysmal positional vertigo (BPPV), eight $(8.9 \%)$ of patients were vestibular neuritis (VN), and eight $(8.9 \%)$ of them were Meniere's disease (MD). $54(60 \%)$ patients were female and $36(40 \%)$ of them were male. $40(44.4 \%)$ of the patients were under 50 years old and $50(55.6 \%)$ patients were older than 50 years. VOR gain loss was detected in 61 of 90 patients $(67.8 \%)$ according to vHIT. Pathological deficiency of VOR for SCCs was detected in 49 of 74 (66.2\%) patients with BPPV, all eight patients with VN (100\%), and four of eight patients with Meniere's disease (50\%) (Table 1). $40(44.4 \%)$ of the patients were under 50 years old and $50(55.6 \%)$ patients were older than 50 years. While SCC pathology was detected in 25 of 36 male $(69.9 \%)$ it was detected in 36 of 54 female $(66.6 \%)$ patients. There was no significant difference between genders for the rate of SCC with VOR gain deficiency $(\mathrm{p}=0,192)$, (Table 2) .

According to caloric stimulation, an average of $54.5+-10.6 \%$ decrease in response was detected in VN patients. VOR gain deficiency was detected in all eight patients with VN. There were three $(37.5 \%)$ patients with both RL and RA involvement (Fig. 2), three (37.5\%) patients with RL, one (12.5\%) patient with RP, and one $(12.5 \%)$ patient with both LL and LP involvement. The average VOR gain value was $0.77+-0.03$ for lateral SCCs and $0.65+-0.11$ for vertical SCCs. VOR gain loss was detected in 4 of eight (50\%) patients with Meniere's disease in vHIT $(\mathrm{RL}=2, \mathrm{LL}=2)$. The average VOR gain value is calculated as $0.66+-0.05$ in pathological lateral SCCs. The average VOR gain value was $0,74+-0,08$ in BPPV, $0.65+-0.11$ in VN, and $0.66+-0.05$ in $\mathrm{MD}(\mathrm{p}=0.253$, Kruskal-Wallis test $)$.

According to physical examination and diagnostic maneuvers, BPPV was most caused by LP SCC ( $=27$, $36.4 \%)$, and at least by RA SCC $(\mathrm{n}=2,2.7 \%)$. According to vHIT, RL SCC (average VOR $=0,76+-0,08$ ) involvement was detected $26(35.1 \%)$ patients with BPPV . LP SCC (average VOR $=0,73+-0,09)$ involvement was detected in $11(14.8 \%)$ patients, and LA SCC (average VOR $=0,3+-0,17)$ involvement was in $4(5.4 \%$ ) patients(Fig. 3) . The sensitivity and specificity calculated as $55.4 \%$ and $81.2 \%$ for all BPPV patients. The highest sensitivity rate was found for LA and RA SCC (100\%), while the highest specificity was calculated for RL SCC (22.5\%). VOR gain deficiency was detected in $15(20.2 \%)$ patients in one or more SCCs in the BPPV group. Both LL and LA SCCs involvement found in 3 (20\%) patients most frequently (Table 3).

\section{Discussion}

Although SCC channel VOR gain values were shown in BPPV patients in the study, demonstrating that SCC VOR gain values returned to normal after medical treatment or canalith repositioning procedures could increase the validity of this study for BPPV. In addition, vHIT protocol performed after making a definite diagnosis with an examination and medical history. Also, the clinician performing the vHIT test was a member of the clinician group conducting the study protocol so this increases the risk of bias since of unfavorable blindness.

The most common cause of vertigo is BPPV and is mostly caused by posterior SCC in this study and as the literature indicates [1]. Although $40 \%$ of BPPV rates originating from lateral SCC have recently been mentioned, this rate is around $20 \%$ in studies with a large number of participants and there is no prevalence study in recent literature $[9,10]$. In some studies, the variability of these rates has been attributed to the missed diagnosis of LSCC-induced BPPV due to the high spontaneous recovery rates [11-13]. Clinically in this study, BPPV was most commonly caused by posterior SCCs (PSCCs) (n=49,66.2\%), and LSCCs involvement were detected in $20(27.2 \%)$ patients. Pathological VOR gain values are detected in $20(27 \%)$ patients for PSCC and in $36(48.6 \%)$ patients for LSCCs. The clinical findings in our study are compatible with the literature, but according to the vHIT test results, LSCCs participation is higher than the rates mentioned in the literature. 
Mahringer et al. [6]. found the sensitivity and specificity as $93 \%$ and $72 \%$ in acute cases $(\mathrm{n}=49$, onset $<5$ days), $59 \%$ and $93 \%$ in other cases $(n=123)$ for vHIT compared to clinical diagnosis. A total of $42(34 \%)$ patients with BPPV were included in this study, and vHIT was found to be positive in $25.7 \%$ of BPPV patients in the acute phase. The sensitivity and specificity for BPPV were not calculated separately [6]. Guan et al. [14] reported that VOR gain values were statistically significantly lower in the acute vertigo group ( $\mathrm{n}=96$, onset $<7$ days), which included 43 BPPV patients, compared to normal participants $(\mathrm{n}=50)$. In this study, VOR gain values were found to be significantly lower in the VN group compared to the acute vertigo group. The sensitivity and specificity for BPPV were not calculated in this study. Additionally, for $\mathrm{VN}$, sensitivity and specificity were reported as $87.9 \%$ and $94.8 \%$ among all patients [14]. In this study sensitivity and specificity found to be $55.4 \%$ and $81.2 \%$ for all BPPV patients. The results are lower than the rates reported in other peripheral vestibular diseases in the literature. Recent literature does not recommend vHIT testing for the diagnosis of BPPV patients, so there are no detailed results specific to BPPV patients and comparative analysis is difficult $[5,7]$. The highest sensitivity rate was found for LA and RA SCC (100\%), while the highest specificity was calculated for RL SCC (22.5\%). However, the reliability of these results is low due to the small number of cases for each SCC.

Blodow et al. [15] detected average VOR gain value as $0,96+-0,08$ on the right and $0.97+-0, .09$ on the left in VHIT. They accepted the VOR gain below 0,79 as pathological for LSCC. The average VOR gain of pathological LSCC was detected by $0.44+-0.20$ in all patients $(n=117)$. This value was detected $0.43+-0.20$ in patients with vestibular neuritis (52) and $0.60+-0.20$ for Meniere's disease $(n=22)$ [15]. In our study, the average VOR gain value was $0,74+-0,08$ in BPPV, $0.65+-0.11$ in VN (LSCC), and $0.66+-0.05$ in MD $(\mathrm{p}=0.253)$. Both accepted threshold and deficient VOR gain for VN and MD similar in analogous studies [16-18]. Miłoński et al. [19] found the rate of 2 or more SCC involvement as $17.8 \%$ in 73 patients with no final diagnosis. Also, these findings are consistent with the rates in our study, VOR gain deficiency was detected in $15(20.2 \%)$ patients in one or more SCCs in the BPPV group.

Clinical findings in this study coincide with the literature in patients with peripheral vertigo, but the vHIT protocol is inconvenient for differentiating patients with a diagnosis of BPPV or determining the specific SCC involvement. Korsager et al. [20] reported abnormal high gain values in vHIT caused by technical error as hand position, head rotation speed of the operator, tightly or loosely fastened device, distance from the wall, unsuitable calibration.

The inconvenience of vHIT test in the diagnosis of BPPV may be due to the reasons listed above, but our final conclusion is parallel with the literature [21]. VOR gains obtained vHIT protocol does not work for BPPV. Also, the clinically detected responsible SCC does not accurately represent by vHIT. In accordance with the literature, vHIT is a fast, simple, and comfortable test in the differential diagnosis of peripheral and central vertigo. The diagnostic value of vHIT in VN and DM has been accepted in the literature. Also, vHIT seems an optimal option for demonstrating peripheral vertigo in patients complaint of acute vertigo, and when supported by clinical information and physical examination, can assist the clinician in the definitive diagnosis.

\section{Conclusion}

Physical examination and medical history are still the first steps in the definitive diagnosis of peripheral vertigo. vHIT is a new protocol and provides objective findings for SCC functions. vHIT can be performed faster and easier than the caloric stimulation test for VN diagnosis, and its results are comparable to the caloric test. However, a negative vHIT test may help early diagnosis of central etiology in patients presenting with acute vertigo. vHIT does not provide sufficient results for BPPV to distinguish from other peripheral vestibular system diseases or does not indicate the involvement of the responsible SCC.

Acknowledgements: None

Data Availability Statement: The data can be shared upon request.

\section{References}


1. Furman JM, Cass SP. Benign paroxysmal positional vertigo. N Engl J Med. 1999;341(21):1590-1596. doi:10.1056/NEJM199911183412107

2. Magnan J, Özgirgin ON, Trabalzini F, et al. European Position Statement on Diagnosis, and Treatment of Meniere's Disease. J Int Adv Otol. 2018;14(2):317-321. doi:10.5152/iao.2018.140818.

3. Lee JY, Park JS, Kim MB. Clinical Characteristics of Acute Vestibular Neuritis According to Involvement Site. Otol Neurotol. 2019;40(6):797-805. doi:10.1097/MAO.0000000000002226.

4. Ulmer E, Chays A. "Head Impulse test de Curthoys \& Halmagyi". Un dispositif d'analyse [Curthoys and Halmagyi Head Impulse test: an analytical device]. Ann Otolaryngol Chir Cervicofac. 2005;122(2):84-90. doi:10.1016/s0003-438x(05)82329-1.

5. Alhabib SF, Saliba I. Video head impulse test: a review of the literature. Eur Arch Otorhinolaryngol. 2017;274(3):1215-1222. doi:10.1007/s00405-016-4157-4.

6. Mahringer A, Rambold HA. Caloric test and video-head-impulse: a study of vertigo/dizziness patients in a community hospital. Eur Arch Otorhinolaryngol. 2014;271(3):463-472. doi:10.1007/s00405-013-2376-5.

7. Welgampola MS, Taylor RL, Halmagyi GM. Video Head Impulse Testing. Adv Otorhinolaryngol. 2019;82:56-66. doi:10.1159/000490272.

8. Basura GJ, Adams ME, Monfared A, et al. Clinical Practice Guideline: Ménière's Disease. Otolaryngol Head Neck Surg. 2020;162(2_suppl):S1-S55. doi:10.1177/0194599820909438.

9. Prokopakis EP, Chimona T, Tsagournisakis M, et al. Benign paroxysmal positional vertigo: 10-year experience in treating 592 patients with canalith repositioning procedure. Laryngoscope. 2005;115(9):1667-1671. doi:10.1097/01.mlg.0000175062.36144.b9.

10. Dai Q, Chen Q, Yin L, Zheng H, Liu SX, Duan M. The long-term follow-up of 61 horizontal canal BPPV after Gufoni and Barbecue maneuver: a prospective study. Acta Otolaryngol. 2020;140(6):463-466. doi:10.1080/00016489.2020.1725114.

11. Parnes LS, Agrawal SK, Atlas J. The diagnosis and management of benign paroxysmal positional vertigo. Semin Hear. 2009;30(4):287-305.

12. Nuti D, Mandalà M, Salerni L. Lateral canal paroxysmal positional vertigo revisited. Ann N Y Acad Sci. 2009;1164:316-323. doi:10.1111/j.1749-6632.2008.03720.x.

13. Singh J, Bhardwaj B. Lateral Semicircular Canal BPPV... Are We Still Ignorant?. Indian J Otolaryngol Head Neck Surg. 2020;72(2):175-183. doi:10.1007/s12070-019-01737-4.

14. Guan Q, Zhang L, Hong W, et al. Video Head Impulse Test for Early Diagnosis of Vestibular Neuritis Among Acute Vertigo. Can J Neurol Sci. 2017;44(5):556-561. doi:10.1017/cjn.2017.202.

15. Blödow A, Pannasch S, Walther LE. Detection of isolated covert saccades with the video head impulse test in peripheral vestibular disorders. Auris Nasus Larynx. 2013;40(4):348-351. doi:10.1016/j.anl.2012.11.002.

16. Blödow A, Heinze M, Bloching MB, von Brevern M, Radtke A, Lempert T. Caloric stimulation and videohead impulse testing in Ménière's disease and vestibular migraine. Acta Otolaryngol. 2014;134(12):1239-1244. doi:10.3109/00016489.2014.939300.

17. Jung J, Suh MJ, Kim SH. Discrepancies between video head impulse and caloric tests in patients with enlarged vestibular aqueduct. Laryngoscope. 2017;127(4):921-926. doi:10.1002/lary.26122.

18. MacDougall HG, Weber KP, McGarvie LA, Halmagyi GM, Curthoys IS. The video head impulse test: diagnostic accuracy in peripheral vestibulopathy. Neurology. 2009;73(14):1134-1141. doi:10.1212/WNL.0b013e3181bacf85 
19. Miłoński J, Pietkiewicz P, Bielińska M, Kuśmierczyk K, Olszewski J. The use of videonystagmography head impulse test (VHIT) in the diagnostics of semicircular canal injuries in patients with vertigo. Int $\mathrm{J}$ Occup Med Environ Health. 2014;27(4):583-590. doi:10.2478/s13382-014-0278-4

20. Korsager LEH, Schmidt JH, Faber C, Wanscher JH. Reliability and comparison of gain values with occurrence of saccades in the EyeSeeCam video head impulse test (vHIT). Eur Arch Otorhinolaryngol. 2016;273(12):4273-4279. doi:10.1007/s00405-016-4183-2.

21. Stevens MN, Garrison DB, Kaylie DM. What is the potential clinical utility of vHIT when assessing adult patients with dizziness?. Laryngoscope. 2017;127(12):2689-2690. doi:10.1002/lary.26774

Table 1 Pathological SCCs involvements according to patient groups

\begin{tabular}{llllll}
\hline & Pathological VOR & Normal VOR & Total & Average VOR Gain* & $\mathbf{p}^{+}$ \\
\hline BPPV & 49 & 25 & 74 & $0,76 \pm 0,08$ & $\mathbf{0 . 2 5 3}$ \\
Vestibular Neuritis & 8 & 0 & 8 & $0.71 \pm 0.11$ & \\
Meniere's Disease & 4 & 4 & 8 & $0.66 \pm 0.05$ & \\
\hline
\end{tabular}

BPPV: Benign paroxysmal positional vertigo VOR: Vestibulo ocular reflex: SCC: Semicircular canal.

${ }^{*}$ Calculated for pathological SCC.

${ }^{+}$Kruskal-Wallis test

Table 2 Pathological semicircular canals involvement according to sex and age groups.

\begin{tabular}{|c|c|c|c|c|c|}
\hline & & Pathological VOR & Normal VOR & Total & $\mathrm{p}$ \\
\hline \multirow[t]{3}{*}{ Female } & Age [?] 50 & 22 & 7 & 29 & 0.289 \\
\hline & Age $>50$ years & 14 & 11 & 25 & \\
\hline & Total & 36 & 18 & 54 & \\
\hline \multirow[t]{3}{*}{ Male } & Age [?] 50 & 9 & 2 & 11 & 0.501 \\
\hline & Age $>50$ & 16 & 9 & 25 & \\
\hline & Total & 25 & 11 & 36 & \\
\hline \multirow[t]{3}{*}{ Total } & Age [?] 50 & 31 & 9 & 40 & \\
\hline & Age $>50$ & 30 & 20 & 50 & \\
\hline & Total & 61 & 29 & 90 & \\
\hline $\mathrm{p}=0.192$ & & & & & \\
\hline
\end{tabular}

VOR: Vestibulo ocular reflex

Table 3 SCCs involvement according to clinical examination and vHIT test for BPPV

\begin{tabular}{lllllll}
\hline & RL & LL & LA & RP & RA & LP \\
\hline Clinical & 13 & 7 & 3 & 22 & 2 & 27 \\
vHIT & 26 & 10 & 4 & 9 & 5 & 11 \\
VOR Gain & $0.76 \pm 0.08$ & $0.73 \pm 0.09$ & $0.3 \pm 0.17$ & $0.47 \pm 0.17$ & $0.42 \pm 0.17$ & $0.15 \pm 0.15$ \\
Sensitivity \% & 92.3 & 85.7 & 100 & 36.3 & 100 & 37.0 \\
Specificity \% & 22.5 & 5.9 & 1.4 & 1.9 & 4.1 & 2.1 \\
\hline
\end{tabular}

SCCs: Semicircular canals, RL: Right Lateral, LL: Left Lateral, LA: Left Anterior, RP: Right Posterior , 
RA: Right Anterior, LP: Left Posterior, vHIT: Video head impulse test VOR: Vestibulo ocular reflex, BPPV: Benign paroxysmal positional vertigo.

\section{Figures}

Figure 1 Video head impulse test report of all semicircular canals in normal values.

Figure 2 Report of deficient vestibulo-ocular reflex gain for right lateral and right anterior semicircular canal due to Vestibular neuritis.

Figure 3 Report of deficient vestibulo-ocular reflex gain for right lateral semicircular canal.
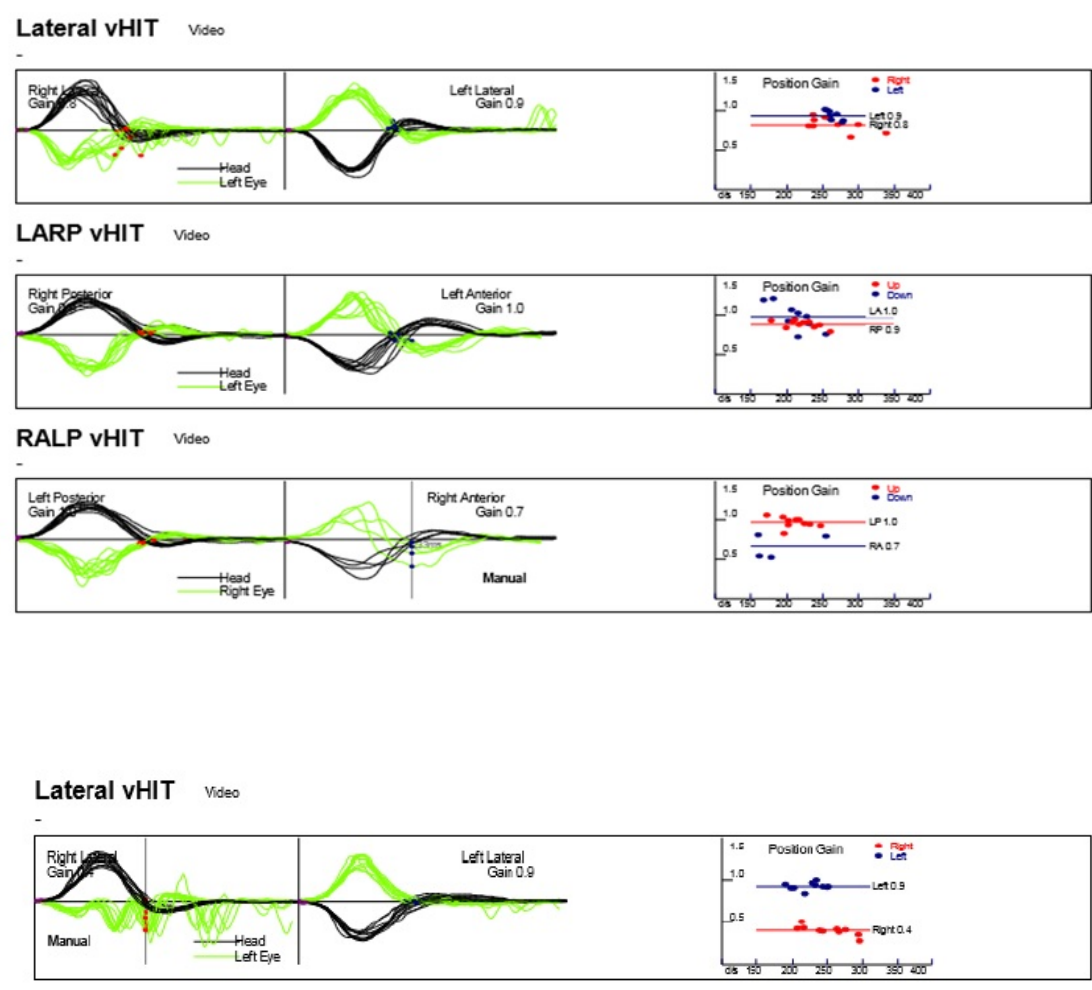

RALP vHIT Video

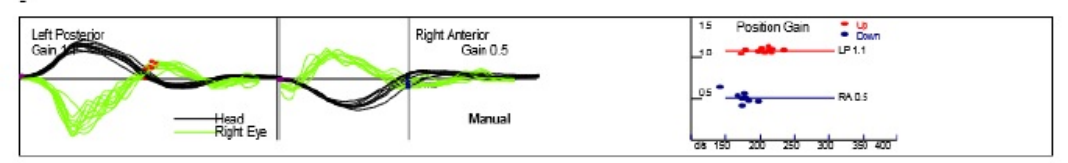

Repeat LARP VHIT video
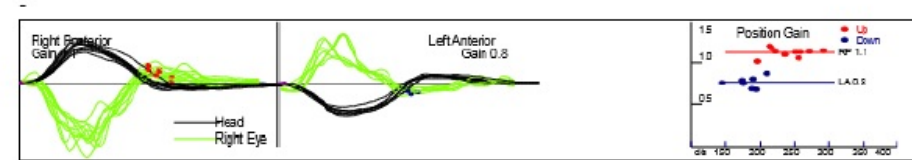
Lateral vHIT Video

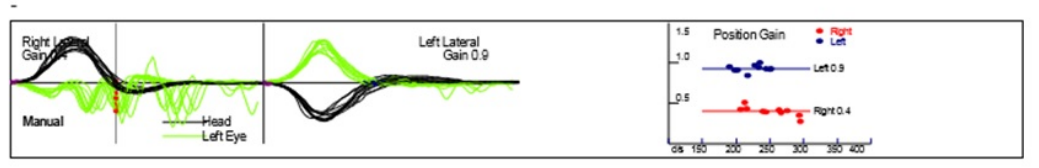

